'Fundación World-Chile. ${ }^{2}$ Comisión Médica Regional Metropolitana Sur. ${ }^{3}$ Instituto Nacional del Cáncer e Instituto de Seguridad del Trabajo.

${ }^{4}$ Comisión Médica Regiona Metropolitana Sur.

Trabajo no recibió financiamiento. Los autores declaran no tener conflictos de interés.

Recibido el 5 de abril de 2021, aceptado el 10 de junio de 2021.

Correspondencia a: Pablo Marcone El Bosque 1913, dpto. 22, Providencia, Santiago pablomarc1@yahoo.com

\section{Reflexión sobre la equidad del sistema de calificación médica para invalidez en Chile}

\author{
ELIANA GORDILLO ${ }^{1}$, PABLO MARCONE ${ }^{2}$, \\ PAOLA RITTER ${ }^{3}$, JORGE VEAS ${ }^{4}$
}

\section{Reflection on the equity of the disability qualification system in Chile}

The Disability Qualification System in Chile and its regulations have inequities as they are applied in a general way and do not consider the peculiar characteristics of vulnerable populations. These people, who are mostly unprotected, have less access to health care levels and receive a lower quality of services. The authors, using a clinical case, we analyze the three main obstacles to obtain a disability pension. A change in applicants' evaluation, considering a broader range of information on the process, a greater symmetry in the evaluated parameters, including biopsychosocial aspects and a better interrelation between Health Services and the Evaluation System would narrow the existing gap between vulnerable and non-vulnerable populations.

(Rev Med Chile 2021; 149: 906-912)

Key words: Disability Evaluation; Insurance, Disability; International Classification of Functioning, Disability and Health; Workers' Compensation.

\section{"La justicia es la ausencia de desigualdades arbitrarias"}

(John Rawls, 1971).

4 1 Sistema de Seguridad Social en Chile está constituido por: Sistema de Salud, Sistema de Pensiones, Seguro de Accidentes del Trabajo y Enfermedades Profesionales y Seguro de Cesantía. El Sistema de Pensiones se ocupa de las contingencias de Vejez, Invalidez y Muerte ${ }^{1}$.

En este escrito se pregunta si la forma en que está constituido el sistema de calificación de invalidez chileno guarda relación con el respeto de la equidad en lo referente a la seguridad social especialmente de las personas vulnerables ${ }^{2}$. El presente trabajo utiliza la palabra "invalidez" definida en la Norma Técnica del DL 3500/1980, como sinónimo de menoscabo laboral, y no se refiere a "persona con discapacidad" definida en el decreto 47 de Ley $20.422^{3}$.

\section{Antecedentes del proceso de calificación médica de invalidez}

La calificación médica de la invalidez es un proceso que evalúa la pérdida, limitación o restricción para el desarrollo de las Actividades de la Vida Diaria (AVD). Tal pérdida se denomina "menoscabo laboral", cuya evaluación se rige por las Normas de Evaluación y Calificación Laboral del Grado de Invalidez de los trabajadores afiliados al nuevo sistema previsional o Norma Técnica en Chile $(\mathrm{NT})^{4}$. La Superintendencia de Pensiones (SP), es el organismo controlador que fiscaliza y regula el sistema de pensiones, incluyendo a Administradoras de Fondos de Pensiones (AFP), Compañías Aseguradoras (CA), Comisiones Medicas Regionales de Evaluadoras de Invalidez $(\mathrm{CMR})^{5}$. En el libro III Beneficios Previsionales del DL $3500^{6}$ se detalla el funcionamiento de las Comisiones Médicas Regionales (CMR), que son 
las responsables de realizar la calificación de menoscabo laboral a:

1. Afiliados y sus beneficiarios de AFP según lo establece el Decreto Ley N ${ }^{\circ} 3.500$.

2. Solicitantes de Pensión Básica Solidaria de Invalidez (PBSI) del Instituto de Previsión Social (IPS).

3. Beneficiarios de Licencia Médica y Subsidios de Incapacidad Laboral Temporal, Ley $\mathrm{N}^{\circ} 20.585$ del 2012 del MINSAL pertenecientes al Fondo Nacional de Salud (FONASA) e Instituciones de Salud Previsional (ISAPRE), que presentan solicitudes de evaluación por la sospecha de enfermedad irrecuperable.

Las CMR son administradas por la Fundación de Administraciones de Comisiones Médicas (FACM), creada por las AFP. Por tanto, el proceso de evaluación del menoscabo laboral tiene una interacción o vínculo público-privado. Las CMR están conformadas por médicos evaluadores de menoscabo laboral con distintos roles que garantizan la calidad técnica de la evaluación ${ }^{7}$ (Tabla 1), un médico Presidente Coordinador Regional, encargado de gestionar las actividades médicas, y un Jefe Administrativo de la gestión sobre los colaboradores. La NT es elaborada por una por una Comisión Técnica de Invalidez $(\mathrm{CTI})^{8}$, de la cual no forman parte los médicos que evalúan menoscabo laboral.

Acorde al lenguaje utilizado en las $\mathrm{NT}^{4}$ se denomina Impedimento a "la enfermedad, alteración, pérdida, evidencia médica, discapacidad o debilitamiento de las fuerzas físicas o intelectuales que afecta la capacidad de trabajo". Es considerado como Configurado cuando cumple los requisitos de ser: objetivable, demostrable, tiene medidas generales y terapias médicas o quirúrgicas accesibles, evolución en estabilización o agravación y cumple lo períodos de observación clínica. Los impedimentos configurados son sometidos al cálculo de suma combinada, diferente a la suma aritmética. El resultado de esta suma es el menoscabo laboral, definido como la perdida, limitación o restricción para el desarrollo de las Actividades de la vida Diaria” (AVD). Estas últimas las clasifican en esenciales, domésticas, de desplazamiento y sociales, en un listado no estructurado ${ }^{4}$.

En las CMR son evaluados los solicitantes de pensión de invalidez y consignados los impedimentos acordes a la NT vigente. El médico asignado al caso, realiza una valoración clínica presencial del solicitante, pudiendo requerir peritajes externos y entrega una recomendación a la sesión de CMR respecto a la calificación de la invalidez. Los peritajes externos son realizados por médicos u otros profesionales especialistas denominados "interconsultores" y/o instituciones dedicadas a exámenes complementarios. Todos ellos acreditados ante la $\mathrm{SP}^{6}$.

La Sesión de CMR tiene como objeto revisar la solicitud y emitir un Dictamen Correcto, cuyas conclusiones posibles son: rechazo de pensión, pensión total o pensión parcial. El plazo legal para la emisión del primer dictamen es de 60 días desde la evaluación médica y puede ser prorrogable por 60 días más. Este dictamen puede ser apelado por el solicitante o la CA iniciando una segunda revisión en la Comisión Médica Central (CMC). Esta, en base a datos del expediente y la NT, define si el caso estuvo correctamente evaluado y rechaza la apelación ratificando el dictamen o, incorrectamente evaluado solicitando peritajes para resolver posteriormente con los nuevos antecedentes ${ }^{6}$.

Tabla 1. Características generales de Médicos integrantes de CMR

\begin{tabular}{|lcccc|}
\hline Cargo médico & Dependencia & $\begin{array}{c}\text { Derechos sesión de comisión } \\
\text { Voz }\end{array}$ & $\begin{array}{c}\text { Evaluación presencial de } \\
\text { pacientes }\end{array}$ \\
\hline Presidente & SP & Sí & Sí & Sí \\
Secretario & SP & Sí & Sí & Sí \\
\hline Integrante & SP & Sí & Sí & Sí \\
Médico asesor de afiliado & SP & Sí & No & Sí \\
\hline Médico observador & SA & Sí & No & No \\
\hline
\end{tabular}

*Solamente evalúa a solicitantes cubiertos por Seguro de Invalidez y Sobrevivencia (SIS). 


\section{Presentación de caso clínico}

Se presenta como ejemplo, un caso clínico que permite hacer una reflexión sobre la equidad del sistema de calificación médica para la invalidez en Chile.

J.P.P., tiene 57 años, anteriormente albañil con trabajo ocasional en ferias libres, recibe la sospecha diagnóstica de gonartrosis severa por su médico de Atención Primaria de Salud (APS), tiene dificultades para ir al baño y sentarse en el inodoro, le cuesta ponerse el pantalón y los zapatos, no tiene una vivienda adaptada, vive con su esposa quien trabaja todo el día. Se presentó a trámite de pensión de invalidez y desde la Comisión Médica fue enviado a interconsulta de traumatología quien le solicitó una radiografía de rodillas. Meses después, llegó a su domicilio una carta con la "resolución", de la que solamente comprendió la palabra "Rechazado"; lo de Impedimento No Configurado no supo a qué se refería. JPP ya no puede trabajar, se siente una carga, su esposa lo acompaña a la CMR a preguntar qué significa la carta. Una funcionaria administrativa le explica que puede presentar una apelación a la CMC, y dado que no cuenta con medico asesor la escribe a mano: "ya no puedo trabajar y el tratamiento no me sirve". En dos meses le llega la respuesta: "Se aplicó correctamente la Norma, no se acoge el reclamo, se rechaza la invalidez". Entonces JPP se siente impotente ante sus evaluadores.

La historia de JPP motiva el análisis del proceso de evaluación de menoscabo laboral desde la perspectiva del solicitante. Para ello, se realizó una búsqueda, de literatura comparada y de antecedentes políticos y, además, se reflexionó sobre ¿cuáles son los nodos de una correcta y equitativa evaluación de menoscabo laboral?

\section{Antecedentes en la literatura comparada}

\section{Perfil de los solicitantes de pensión de invalidez}

Un estudio colombiano, cuyo objetivo fue caracterizar la reclamación y aprobación de pensiones de invalidez por 5 años ${ }^{9}$, señala que los factores que explican el $41,0 \%$ de la probabilidad de revertir un rechazo de una solicitud de pensión de invalidez son la edad del afiliado entre 30 y 34 años, tener estudios universitarios, además de las causales de solicitud. No hay datos chilenos publicados sobre la relación entre el nivel de menoscabo laboral con la escolaridad, la edad y nivel de ingresos económicos. El único trabajo chileno publicado sobre pensiones de invalidez encontrado en buscador Pubmed, es el de Zamora et al. ${ }^{10}$ donde se analizaron bases de datos secundarias del total de las solicitudes de los afiliados a AFP cubiertos por el Seguro de Invalidez y Sobrevivencia (SIS) del período 1990-1992, correspondiente a 13.456 personas. Las causales de presentación en orden decreciente fueron: 1) Osteoarticulares; 2) Cardiovasculares; 3) Psiquiátrica. Del total, el 59,0\% no obtuvo pensión de invalidez, el 31,0\% obtuvo invalidez total y el 10,0\% invalidez parcial. Las principales causas de invalidez total fueron oncológica, neurológica y cardiovascular. Los impedimentos osteoarticulares, pese a ser el primer motivo de presentación, constituyeron la primera causal de rechazo de pensión (Tabla 2).

\section{Instrumentos de medición de invalidez o discapacidad}

La Organización Mundial de la Salud (OMS), desarrolló en el año 2001 la Clasificación Internacional del Funcionamiento, la Discapacidad y la Salud (CIF) que incluye un modelo conceptual y taxonómico del funcionamiento humano ${ }^{11.12}$. En la actualidad, existen instrumentos abreviados de medición de discapacidad basados en CIF, validados y fiables, que permiten asignar un valor a los factores que inciden en la funcionalidad global de la persona ${ }^{13}$. Chang et al., explica el cambio aplicado en Taiwán desde el modelo biomédico de calificación de invalidez a otro que integra el concepto biopsicosocial basado en el uso de instrumento $\mathrm{CIF}^{14}$, que incluye la capacidad de desarrollar actividades, la participación social de los solicitantes y la influencia de los factores ambientales en la discapacidad. En EEUU, fue publicada el año 2008 la sexta edición de The American Medical Asociation (AMA) Guides to the Evaluation of Permanent Impairment ${ }^{15}$, donde se incorporan las definiciones y la terminología CIF y proporcionan un medio sencillo de evaluación de las AVD. Con todo, la NT en Chile al 2020 bajo la cual se clasifica el menoscabo laboral, no se ajusta a lo sugerido por la OMS y AMA en cuanto no evalúa a las personas en la integralidad de su funcionamiento. 
Tabla 2. Causas de presentación y grado de invalidez de afiliados a AFP a calificación de invalidez y resultados, período 1992-1993 (autorizado por Zamora y cols.) ${ }^{11}$

\begin{tabular}{|c|c|c|c|c|c|c|c|c|}
\hline \multirow[t]{2}{*}{ Causales } & \multicolumn{2}{|c|}{ Casos } & \multicolumn{2}{|c|}{ Invalidez total } & \multicolumn{2}{|c|}{ Invalidez Parcial } & \multicolumn{2}{|c|}{ Menor a $\mathbf{5 0} \%$} \\
\hline & $\mathbf{n}$ & $\%$ & $\mathbf{n}$ & $\%$ & $\mathbf{n}$ & $\%$ & $\mathbf{n}$ & $\%$ \\
\hline Osteoarticulares & 4.460 & 33,1 & 247 & 5,5 & 558 & 12,5 & 3.655 & 81,9 \\
\hline Cardiovasculares & 1.863 & 13,8 & 846 & 45,4 & 91 & 4,8 & 926 & 49,7 \\
\hline Psiquiátrica & 1.354 & 10 & 306 & 22,5 & 179 & 13,2 & 869 & 64,1 \\
\hline Oftalmológicas & 1.115 & 8,2 & 412 & 36,9 & 145 & 13,0 & 558 & 50,0 \\
\hline Oneológicas & 1.049 & 7,7 & 809 & 77,1 & 16 & 1,5 & 224 & 21,3 \\
\hline Neurológicas & 997 & 7,4 & 520 & 52,1 & 82 & 8,2 & 395 & 39,6 \\
\hline Respiratoria & 695 & 5,1 & 257 & 36,9 & 65 & 9,3 & 373 & 53,6 \\
\hline Otorrinolaringológicas & 425 & 3,1 & 66 & 15,5 & 56 & 9,3 & 303 & 71,2 \\
\hline Gastroenterológicas & 320 & 2,3 & 92 & 28,7 & 42 & 13,1 & 186 & 58,1 \\
\hline Mesenquimopatías & 236 & 1,7 & 78 & 33 & 46 & 19,4 & 112 & 47,4 \\
\hline Otras patologías & 942 & 7,0 & 525 & 55,7 & 60 & 6,3 & 357 & 37,8 \\
\hline Total & 13.456 & 100 & 4.158 & 30,9 & 1.340 & 9,9 & 7.958 & 59,1 \\
\hline
\end{tabular}

Fuente: Zamora L, Arellano M, Kunstmann S, Montenegro A, Riveros B, Schlegel I, Silva P, Vidal C. Invalidez en el sistema privado de pensiones en Chile [Disability in the private pension system in Chile]. Rev Med Chile. 1997; 125 (1): 99-106.

\section{Sobre los derechos humanos}

La Convención sobre los derechos de las personas con discapacidad, vigente desde el 2008, marcó un cambio de paradigma, de los enfoques tradicionales basados en la caridad, a una estrategia basada en los derechos humanos ${ }^{16}$. La Declaración Universal de los Derechos Humanos incluye prioridad de acceso a los servicios de salud de las comunidades pobres y desfavorecidas ${ }^{17}$. Los grupos vulnerables chilenos evidencian barreras en el acceso a la salud y sus condiciones socioculturales inciden por una parte en peores resultados de salud individual ${ }^{18}$.

\section{Antecedentes políticos}

La Comisión Especial Investigadora de la Cámara Diputados de Chile constituida el 2018, concluyó lo siguiente respecto a la concesión y denegación de pensiones de invalidez y sobrevivencia $^{19}$ :

\section{Insuficiente asesoría médica}

En la reforma previsional del año 2008 se creó el cargo de médico asesor a los solicitantes cubier- tos por el SIS para corregir la asimetría entre solicitante y las $\mathrm{CA}^{6}$. Sin embargo, según un testimonio, el menor porcentaje de los solicitantes cuentan con el respaldo de médico asesor; y se concluye que las horas contratadas de médicos asesores deberían ser acorde al número de solicitantes afiliados al SIS. Otra conclusión fue la necesidad de integrar el trabajo de la Comisión de Medicina Preventiva e Invalidez (COMPIN) y las CMR por el alto porcentaje de rechazo de licencias médicas de los prestadores de salud privados a personas que se encuentran tramitando su pensión de invalidez.

\section{Diagnóstico de nodos críticos}

Según los autores del presente escrito existen nodos en el sistema que inciden negativamente en la equidad del proceso de calificación para la obtención de un Dictamen Correcto de Pensión de Invalidez y estos son:

\section{Insuficiente difusión en el proceso de evaluación de pensión de invalidez}

Este proceso es desconocido por la mayoría de los médicos dado por ejemplo que al 2020 en 
las webside de las Universidades del Consejo de Rectores, no están descritas estas materias en las mallas curriculares de carrera de medicina. Esto a su vez implica que los médicos tratantes no cuentan con la información sobre cuáles son los requisitos que las personas deben cumplir para la obtención del beneficio. Más aún, no existe un mecanismo de retroalimentación entre los médicos tratantes y la CMR con el objetivo de emitir Dictámenes Correctos. Es así que, el uso de un dictamen con siglas y conceptos ajenos al quehacer habitual, enfrenta a los médicos tratantes a un dictamen de difícil interpretación. Por otra parte de la SP no ha existido una política efectiva tendiente a favorecer la difusión sistemática, clara y de fácil acceso sobre la metodología de calificación de invalidez a los profesionales de la salud chilenos. Por ejemplo, los informes de los indicadores de evaluación y gestión de la SP hasta el año 2020 no son de acceso en forma simple para los investigadores del tema como tampoco para los médicos tratantes. Esta insuficiente difusión, puede determinar en parte la presentación de solicitudes infundadas e incompletas que pudieran ir en desmedro de las expectativas de las personas. Puede ocurrir que los solicitantes y sus médicos tratantes esperen que la obtención del beneficio de una pensión de invalidez sea indiscutible y no consideren que serán sometido a un proceso de calificación que determine la negación de una pensión.

\section{Evidencia de asimetría entre el solicitante y el modelo chileno de calificación de invalidez}

En este trabajo la asimetría se define como desigualdad en el acceso a la seguridad social y se clasifica en:

\subsection{Asimetrías previas a la calificación}

Chile es el segundo país de la OCDE con la mayor desigualdad en la distribución del PIB expresado mediante el coeficiente GINI, que se ha mantenido inalterado a lo largo del tiempo $(0,50$ en 2006 y 0,49 el 2017) ${ }^{20}$. La literatura indica que los grupos con peores condiciones socioeconómicas tienen una mayor carga de enfermedad, enfermedades crónicas e incapacidades a edades más tempranas, menos acceso a los servicios de salud y estos son de peor calidad ${ }^{21}$. El empleo de una NT es una medida igualitaria y justa si la población fuera homogénea en acceso a la salud.

\subsection{Asimetrías durante la calificación}

Tal como se explicó en el párrafo de los antecedentes políticos, el médico asesor quien explica, acompaña y eventualmente redacta la apelación de la resolución de invalidez, solo participa si el solicitante tiene cobertura por el SIS. El DL 3500 no considera asesoría médica a los solicitantes sin cobertura salvo que acuda a un Asesor Previsional privado pagado de su bolsillo ${ }^{22}$. Para los que no cuentan con el SIS ni con los medios para el pago de asesoría, y quieren ejercer el derecho de apelación lo harán sin conocimiento de la ley que se les aplicó, con sus propios recursos socioculturales, estableciéndose diferencias insoslayables entre los solicitantes.

Por otro lado, tal y como fue mencionado en el párrafo sobre los DDHH y en el de asimetría previa a la calificación, el desigual acceso a diagnósticos y tratamientos oportunos para las personas vulnerables, establece una asimetría desfavorable respecto a la aplicación de la NT de calificación de invalidez. No son considerados los diagnósticos en el cálculo de menoscabo laboral, cuando no cumplen con los requisitos exigidos de diagnóstico fundado, estar acogido a Garantías explícitas en salud (GES), haber recibido terapias médicas y/o quirúrgicas completas, derivación a centros de mayor complejidad, y quedan registradas en el dictamen bajo la glosa de "No Configurado". Estas acciones de salud incompletas, dependen de la capacidad resolutiva del sistema de salud, y no del solicitante.

Otro aspecto es el uso de un instrumento para la recolección de datos sobre las AVD que no ha sido sometido a un proceso técnico de validez de contenido, por lo que no se asegura que sea fiable, reproducible, ni que recoja los antecedentes suficientes requeridos para la calificación de menoscabo laboral. La no incorporación hasta el año 2020 de un instrumento como los sugeridos por la OMS y la AMA, que considere otras determinantes que afectan directamente las AVD y no solo los aspectos biomédicos, puede influir negativamente en las poblaciones vulnerables para la obtención de una pensión de invalidez.

En suma, la insuficiente difusión de la metodología de calificación de invalidez en Chile, el desigual acceso a la salud, la aplicación de un instrumento que no considera la influencia de los factores ambientales en la discapacidad, y la no existencia de la figura de un médico asesor 
universal, puede afectar la equidad del sistema de calificación de invalidez en Chile.

\section{Deficiente interrelación con otras organizaciones afines}

Se entiende por interrelación, al enlace que debería existir entre entidades afines que participan en el proceso de salud de una persona que solicita una pensión de invalidez. Los distintos niveles de atención de salud, ya sean públicos o privados, y la COMPIN en su tarea de control de las licencias médicas, deberían interrelacionarse estratégicamente con un proceso justo de evaluación de invalidez para el solicitante. Por ejemplo, en atención primaria de salud se aplica el Instrumento de Valoración de Desempeño en Comunidad "IVADEC-CIF"23, que analiza a las personas de manera multidimensional y es un insumo para que COMPIN realice el ingreso al "Registro Nacional de Discapacidad"24. Las CMR no acceden al procedimiento descrito y tampoco a informes médicos por licencias prolongadas.

El DL 3500 establece que solamente en condiciones de salud irrecuperables, corresponde la evaluación de menoscabo laboral por la CMR, pero actualmente producto de las limitaciones en el otorgamiento de prestaciones de recuperación de la salud, pudieran terminar personas con salud recuperable obteniendo el beneficio económico llamado pensión de invalidez.

La evaluación de menoscabo laboral, debiera ser la última de una cadena de acciones tendiente a la mejoría del estado de bienestar.

\section{Conclusiones}

De la reflexión analítica del presente trabajo se concluye que el proceso igualitario que establece una norma técnica de calificación para obtención de pensión de invalidez en Chile, se ve severamente afectado por la falta de difusión del proceso entre los actores tanto por los solicitantes como el personal de la salud, por la asimetría entre el acceso a las prestaciones de salud de las poblaciones vulnerables con las no vulnerables y por la precaria interrelación entre el Sistema de salud y el Sistema Evaluador. Para mejorar la equidad en la evaluación del grado de menoscabo laboral se propone la búsqueda de nuevos instrumentos de valoración (por ejemplo, los basados en el modelo CIF) que permitan la visión integrada del ser humano, como un todo asociado a su comunidad, ambiente, trabajo, y las limitaciones que pueda presentar en estos, más que como un ser individual con deficiencias biomédicas. Como observación final del presente análisis, los autores quieren llamar la atención en que siendo la discapacidad un tema relevante en Seguridad Social, no se encuentren mayores trabajos científicos en Chile publicados del tema, por lo que llaman a desarrollar un proyecto transdisciplinario que incluya a los actores sociales, agrupaciones de pacientes y expertos técnicos para transparentar los procesos, difundir la realidad del menoscabo laboral en Chile y así establecer un nuevo pacto más equitativo con las poblaciones vulnerables.

\section{Referencias}

1. Constitución Política de la República de Chile 1980. Art. 19 El derecho a la vida y a la integridad física y psíquica de la persona. En: Capítulo II de los Derechos y Deberes Constitucionales,. Editorial Jurídica de Chile, tercera edición marzo 2020.

2. Naciones Unidas. Art. 22, Derechos económicos, sociales y culturales de todas las personas para su bienestar. Declaración Universal de Derechos Humanos 1948. https://www.un.org/es/universal-declaration-human-rights/

3. Decreto Ley 47 de Ley 20.422. Reglamento para la Calificación y Certificación de La Discapacidad. Título I, Artículo 4 letra a. https://www.bcn.cl/leychile/navegar?idNorma $=1055217$.

4. Superintendencia de Pensiones de Chile. Normas para la evaluación y calificación del grado de invalidez de los trabajadores afiliados al nuevo sistema previsional. Diario Oficial, Comisión Técnica de Invalidez. Séptima edición. 2016. https://www.spensiones.cl/transparencia/normas $\% 20$ para $\% 201 \mathrm{la} \% 20$ evaluacion $\% 20 \mathrm{y} \% 20$ calificacion $\% 20 \mathrm{de} \% 20$ invalidez $\% 20 \mathrm{de} \% 20$ los $\% 20$ trabajadores\%20afiliados\%20al\%20nuevo\%20sistema $\% 20$ previsional-1.pdf

5. Ley 20.255 del 1 de marzo del 2008. Superintendencia de Pensiones. https://www.spensiones.cl/portal/institucional/594/w3-article-7330.html\#: :text=Crea $\% 20 y \% 20$ establece\%20un\%20Sistema,APS)\%20de\%20vejez\%20 $\mathrm{e} \% 20$ invalidez.

6. Superintendencia de Pensiones de Chile. Título XIII Archivo centralizado de expedientes de calificación de invalidez. En: Compendio de Normas del Sistema de 
Pensiones. Libro III Beneficios Previsionales, número 2, letra k. https://www.spensiones.cl/portal/compendio/596/w3-propertyvalue-2816.html

7. Superintendencia de Pensiones de Chile. Comisiones médicas. https://www.spensiones.cl/portal/institucional/594/w3-propertyvalue-9983.html.

8. Ministerio del Trabajo y Previsión Social. Ley Núm. 18964, Publicada el 10 de marzo 1990. Modifica El Decreto Ley $\mathrm{N}^{\circ} 3.500$, de 1980 y las Leyes $\mathrm{N}^{\circ} 18.398$, 18.646, 18.933 Y 18.768. https://legislacion-oficial.vlex. cl/vid/ley-modifica-decreto-leyes-470690754.

9. Yepes-Delgado CE, Henao-Nieto DE, Montoya-Jaramillo M, Montoya-Echeverry L. Caracterización de factores relacionados con la reclamación y aprobación de pensiones de invalidez por enfermedad común en población trabajadora colombiana entre 2006-2011. Iatreia. 2018;31(3): 248-261. DOI 10.17533/udea.iatreia. v31n3a03.

10. Zamora L, Arellano M, Kunstmann S, Montenegro A, Riveros B, Schlegel I, et al. Invalidez en el sistema privado de pensiones en Chile. Rev Med Chile 1997; 125 (1): 99-106. PMID: 9336077.

11. Cieza A, Brockow T, Ewert T, Amman E, Kollerits B, Chatterji S, et al. Linking health-status measurements to the international classification of functioning, disability and health. J Rehabil Med. 2002;34(5):205-10. DOI: $10.1080 / 165019702760279189$.

12. Gutenbrunner C, Meyer T, Melvin J, Stucki G. Towards a conceptual description of Physical and Rehabilitation Medicine. J Rehabil Med. 2011;43(9):760-4. DOI: 10.2340/16501977-0866.

13. Stucki G, Pollock A, Engkasan JP, Selb M. How to use the International Classification of Functioning, Disability and Health as a reference system for comparative evaluation and standardized reporting of rehabilitation interventions. Eur J Phys Rehabil Med 2019;55:384-94. DOI: 10.23736/S1973-9087.19.05808-8).

14. Kwang-Hwa Chang, Wen-Chou Chi, Shih-Wei Huang, Feng-Hang Chang, Hua-Fang Liao, Reuben Escorpizo et al. Perceptions and attitudes towards the implementation of a disability evaluation system based on the international classification of functioning, disability, and health among people with disabilities in Taiwan, Disability and Rehabilitation. 2019; 41 (13): 1552-1560.
DOI: $10.1080 / 09638288.2018 .1442506$

15. Rondinelli RD. Changes for the new AMA Guides to impairment ratings, 6th Edition: implications and applications for physician disability evaluations. PM R. 2009;1(7):643-56. DOI: 10.1016/j.pmrj.2009.04.012.

16. Naciones Unidas. Derechos humanos de las personas con discapacidad. Naciones Unidas, Derechos Humanos, Oficina de Alto comisionado. https://www.ohchr. org/SP/Issues/Disability/Pages/DisabilityIndex.aspx.

17. United Nations. CESCR General Comment No. 14: The Right to the Highest Attainable Standard of Health (Art. 12). Committee on Economic, Social and Cultural Rights. U.N. Doc. E/C.12/2000/4, 18-27. https://www. refworld.org/pdfid/4538838d0.pdf.

18. Vásquez F, Paraje G, Estay M. Income-related inequality in health and health care utilization in Chile, 2000-2009. Rev Panam Salud Publica. 2013;33(2): 98-106. https:// www.scielosp.org/article/rpsp/2013.v33n2/98-106/en/

19. Informe de la Comisión Especial Investigadora de Los Actos de Gobierno, de los Ministerios del Trabajo y de Salud y La Superintendencia de Pensiones, desde el año 2008 a la fecha, en relación con la Concesión y Denegación de Pensiones de Invalidez y Sobrevivencia. https:// www.camara.cl/verDoc.aspx?prmID=48329\&prmTipo=INFORME_COMISION.

20. OECD. National population distribution (indicator). OECD Library 2019. DOI: 10.1787/7314f74f-en.

21. Schneider MC, Castillo-Salgado C, Bacallao J, Loyola E, Mujica O, Vidaurre M. Métodos de medición de las desigualdades de salud. Rev Panam Salud Publica/Pan Am J Public Health 12 (6), 2002. https://scielosp.org/ pdf/rpsp/2002.v12n6/398-414/es

22. Superintendencia de Valores y Seguros. Portal de educación financiera. ¿qué es un asesor previsional?. https:// www.svs.cl/educa/602/w3-article-1525.html.

23. Guajardo A, Recabarren E, Parraguez V. Rehabilitación de base comunitaria. Diálogos, reflexiones y prácticas en Chile. Publicación Universidad Andrés Bello. Primera Edición, Santiago de Chile. 2014, pp:47. https://issuu. com/unab/docs/rbc_en_chile.

24. Servicio Chile Atiende. Certificado de inscripción en el Registro Nacional de la Discapacidad. 2020. https:// www.chileatiende.gob.cl/fichas/3349-certificado-de-inscripcion-en-el-registro-nacional-de-la-discapacidad. 